\title{
Chronic Traumatic Encephalopathy: Is Latency in Symptom Onset Explained by Tau Propagation?
}

\author{
Joshua Kriegel, ${ }^{1,2}$ Zachary Papadopoulos, ${ }^{1,2}$ and Ann C. McKee ${ }^{1,2,3,4,5}$ \\ ${ }^{1}$ Alzheimer's Disease and CTE Center, Boston University School of Medicine, Boston, Massachusetts 02118 \\ ${ }^{2}$ Department of Neurology, Boston University School of Medicine, Boston, Massachusetts 02118 \\ ${ }^{3}$ VA Boston Healthcare System, U.S. Department of Veterans Affairs, Jamaica Plain, Massachusetts 02130 \\ ${ }^{4}$ Department of Veterans Affairs Medical Center, Bedford, Massachusetts 01730 \\ ${ }^{5}$ Department of Pathology, Boston University School of Medicine, Boston, Massachusetts 02118 \\ Correspondence: amckee@bu.edu
}

Chronic traumatic encephalopathy (CTE) is a neurodegenerative tauopathy associated with repetitive mild brain trauma. CTE, previously termed "dementia pugilistica," has been identified in American football, ice hockey, baseball, rugby and soccer players, boxers, wrestlers, and military personnel exposed to blast and other traumatic brain injuries. There is often a long latency period between an individual's exposure to repetitive brain trauma and the clinical symptoms of CTE. The pathology of CTE is characterized by a progression from isolated focal perivascular hyperphosphorylated tau lesions in the cerebral cortex to a widespread tauopathy that involves diffuse cortical and medial temporal lobe regions. We hypothesize that the spread of tau from focal perivascular lesions to a widespread tauopathy occurs as a result of intraneuronal and intrasynaptic prion-like protein templating, as well as tau secretion and propagation along glymphatic and cerebrospinal fluid pathways.

C. hronic traumatic encephalopathy (CTE) is a neurodegenerative tauopathy associated with repetitive mild brain trauma, including concussion and subconcussion. CTE was first recognized under the name "punch-drunk syndrome" and later as "dementia pugilistica." To date, CTE has been identified in American football, ice hockey, baseball, rugby and soccer players, boxers, wrestlers, and military personnel exposed to blast and other traumatic brain injuries. Like many other neurodegenerative diseases, CTE can only be definitively diagnosed during life, and little is known about the incidence and prevalence of the disease. Over the past decade, tremendous advances have been made in the classification and characterization of the clinical and neuropathological features of CTE. Recent biochemical and immunohistochemical studies have shown that the hyperphosphorylated ( $\mathrm{p}$-tau) pathology in CTE differs from Alzheimer's disease (AD) (Kondo et al. 2015; Kanaan et al. 2016). The pathological criteria for diagnosis, as well as a staging scheme for grading pathological severity, have been de-

Editor: Stanley B. Prusiner

Additional Perspectives on Prion Diseases available at www.perspectivesinmedicine.org

Copyright (C) 2018 Cold Spring Harbor Laboratory Press; all rights reserved; doi: 10.1101/cshperspect.a024059

Cite this article as Cold Spring Harb Perspect Med 2018;8:a024059 
J. Kriegel et al.

fined (McKee et al. 2013, 2016). Large-scale clinicopathological studies (Mez et al. 2015) and longitudinal, prospective clinical studies are currently underway to identify clinical criteria for diagnosis, with high sensitivity and specificity from other disorders. Despite these advances, a pressing question remains regarding the pathogenesis of CTE: Why is there usually a prolonged interval of years to decades between an individual's exposure to brain trauma and the onset of clinical symptoms? Some of the possible mechanisms to explain this slow spread of p-tau, which extends from isolated, focal perivascular lesions in the cerebral cortex to a widespread tauopathy that affects many brain regions in the absence of further trauma, include a prion-like mechanism of tau protein templating, tau secretion into the interstitial and cerebrospinal fluid (CSF) spaces, and tau spread along glymphatic channels and CSF pathways, including the Virchow-Robin space surrounding penetrating blood vessels (Iliff et al. 2014).

\section{NEUROPATHOLOGY}

In 1928, the pathologist and medical examiner Harrison Stanford Martland introduced the term "punch drunk" to describe the clinical features of a distinct neuropsychiatric syndrome that affected boxers (Martland 1928), a condition that later came to be known as "dementia pugilistica” (Millspaugh 1937). Case reports describing the neuropathological features of CTE appeared in the 1950s and 1960s (Brandenburg and Hallervorden 1954; Grahmann and Ule 1957; Neubuerger et al. 1959; Courville 1962; Mawdsley and Ferguson 1963; Payne 1968). The characteristic pathological findings included cerebral atrophy, neuronal loss, and silverpositive neurofibrillary tangles (NFTs). In 1973, Corsellis and colleagues described the clinicopathological findings in 15 boxers with a high index of suspicion for "dementia pugilistica” (Corsellis et al. 1973). They noted cerebral atrophy, enlargement of the lateral and third ventricles, thinning of the corpus callosum, cavum septum pellucidum with fenestrations, cerebellar scarring, and silver-positive neurofi- brillary degeneration of the cerebral cortex and substantia nigra (Corsellis et al. 1973).

In the late 1990s, in association with the advent of p-tau immunohistochemistry, Hof et al. (1991) first reported the distinctive pathology of early CTE in young subjects. A 24-year-old autistic woman who exhibited head-banging behaviors was found to have numerous perivascular clusters of neurofibrillary tangles (NFTs) and neurites at the depths of the cerebral sulci and in the superficial cerebral layers of the inferior temporal, entorhinal, and perirhinal cortices in the absence of beta amyloid (A $\beta$ ) (Hof et al. 1991). Geddes and colleagues (Geddes et al. 1996) also described patchy, perivascular deposits of $\mathrm{p}$-tau in the brain of a 23-year-old boxer and commented on the notable absence of NFTs in the medial temporal lobe, a site of early NFT deposition in Alzheimer's disease (AD). Geddes and colleagues further compared the immunohistochemical findings of the five young men, ranging in age from 23-28 years (mean 26 years), exposed to repetitive brain trauma to 21 control subjects. This study described argyrophilic, tauimmunopositive neocortical NFTs and neuropil threads strikingly arranged in groups around small intracortical blood vessels (Geddes et al. 1999). Notably, the hippocampus was normal in all of the cases. Moreover, perivascular tau pathology was found in none of the 21 agematched controls (Geddes et al. 1999). In 2009, McKee and colleagues reviewed all 47 neuropathologically verified cases of CTE reported in the world's literature since 1954 and comprehensively detailed the immunohistochemical characteristics and neuroanatomical regions involved in two former boxers and an American football player (McKee et al. 2009). The investigators emphasized the prominent perivascular pattern of NFTs, the clustering of the pathology at the depths of the sulci, and noted the additional features of p-tau containing astrocytes, and spindle-shaped and dot-like neurites (McKee et al. 2009). The investigators also showed the widespread anatomic involvement in CTE, a p-tau pathology that affects the olfactory bulbs, subcortical U-fibers, diencephalon, nucleus basalis of Meynert, the brain- 

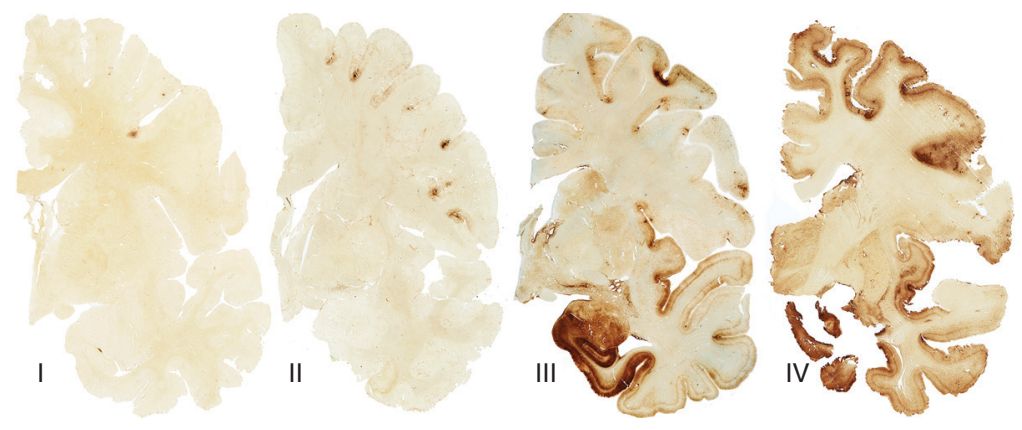

Figure 1. The four stages of chronic traumatic encephalopathy (CTE). In stage I CTE, isolated perivascular p-tau lesions are found in the cerebral cortex. In stage II CTE, multiple perivascular p-tau foci are found in the cerebral cortex. In the adjacent cortex, neurofibrillary tangles (NFTs) are scattered throughout the superficial cerebral layers. In stage III CTE, confluent patches of NFTs, neurites, and p-tau filled astrocytes are found around blood vessels at the sulcal depths and widely distributed throughout the cerebral cortex, including the medial temporal lobe. By stage IV CTE, p-tau pathology is densely distributed throughout the cerebrum, medial temporal lobe structures, brainstem, cerebellum, and occasionally, the spinal cord. Whole-mount sections of $50 \mu \mathrm{m}$ are immunostained with CP-13.

stem, and limbic cortex with sparing of the primary visual cortex. McKee and colleagues also noted that the preferential involvement of the superficial cortical layers was a feature most prominent in the temporal lobe (McKee et al. 2009). The investigators further observed that this pattern of p-tau deposits was unique and could be distinguished from all other known tauopathies, including aging, $\mathrm{AD}$, and frontotemporal lobar degeneration (McKee et al. 2009, 2010).

Subsequent clinicopathological series of CTE were reported in athletes who played a wide variety of contact sports, including American football, soccer, baseball, and ice hockey (Omalu et al. 2011; Goldstein et al. 2012; McKee et al. 2013, 2014; McKee and Robinson 2014). A series of young military veterans exposed to blast injury from improvised explosive devices were also found to have changes of early CTE consisting of focal perivascular clusters of p-tau NFT and neurites, as well as perivascular axonal injury, reactive astrocytosis, and microgliosis (Omalu et al. 2011; Goldstein et al. 2012). In 2013, McKee and colleagues described the spectrum of p-tau pathology in the largest case series of CTE to date, which consisted of 68 male subjects with CTE ranging in age from 17 to 98 years (mean 59.5 years), and compared the find- ings to 18 age-matched controls. In young subjects with the mildest forms of CTE, focal perivascular epicenters of NFTs and astrocytic tangles were found clustered at the depths of the neocortical sulci. In subjects with advanced disease, a severe tauopathy affected widespread brain regions (McKee et al. 2013). Other abnormalities included abnormal deposits of phosphorylated 43-kDa TAR DNA-binding protein (TDP-43) (80\% of cases) that occasionally colocalized with p-tau, axonal dystrophy, and neuroinflammation (McKee et al. 2010, 2013). McKee and colleagues went on to propose preliminary criteria for the neuropathological diagnosis of CTE, as well as a staging scheme of progressive p-tau pathology, consisting of four stages (stages I-IV) (Fig. 1) (McKee et al. 2013). In stage I CTE, isolated perivascular p-tau lesions are found in the cerebral cortex (Fig. 2). In stage II CTE, multiple perivascular p-tau foci are found in the cerebral cortex-in the frontal, temporal, and parietal lobes and insula. In the cortex adjacent to the focal epicenters, NFTs are scattered throughout the cerebral layers, with a tendency to be most numerous in the superficial cortical laminae. Stage III CTE is distinguished from stages I and II by neurofibrillary degeneration in the medial temporal lobe, affecting the hippocampus, entorhinal cortex, 
J. Kriegel et al.

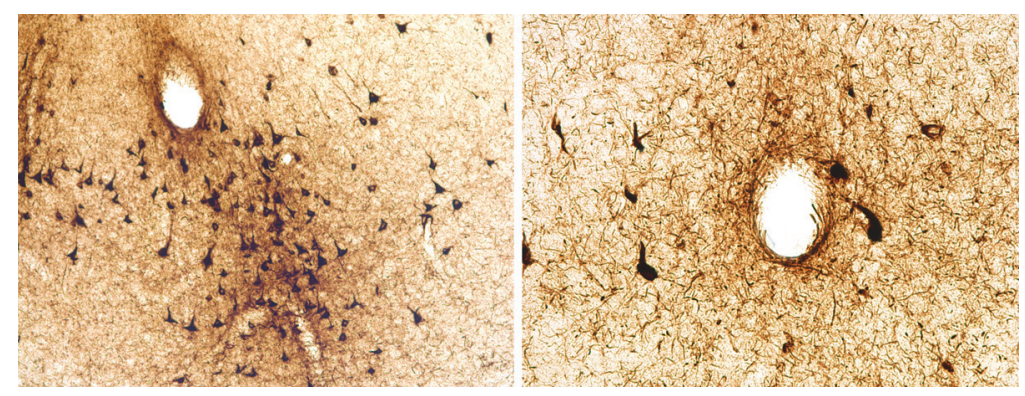

Figure 2. The pathognomonic lesion of CTE. The pathognomonic lesion of CTE consists of an abnormal accumulation of p-tau in neurons, astrocytes, and dot-like structures around small vessels irregularly distributed at the depths of the cortical sulci. Both images are paraffin-embedded, $10 \mu \mathrm{m}$ sections stained with AT8, and are magnified at $400 \times$.

and amygdala. There are also confluent patches of NFTs, neurites, and p-tau-filled astrocytes around blood vessels at the sulcal depths, as well as in linear arrays in the superficial laminae of the cortex. NFTs are typically found in the superior, dorsolateral, and inferior frontal, septal, insular, temporal pole, superior, middle, and inferior temporal and inferior parietal cortices. NFTs are also found in the olfactory bulbs, hypothalamus, mammillary bodies, nucleus basalis of Meynert, substantia nigra, dorsal and median raphe nuclei, and locus coeruleus. By stage IV CTE, severe p-tau pathology is densely distributed throughout the cerebrum, medial temporal lobe structures, brainstem, the cerebellum, and occasionally, the spinal cord (for review, see McKee et al. 2015). Among football players, the stages of CTE pathology are significantly associated with age at death, cumulative years of exposure to football, and interval between retirement from football and death, supporting a dose-response between p-tau deposition and years of playing football, as well as progressive spread of $\mathrm{p}$-tau pathology after football ends.

As a first step toward validating the preliminary McKee criteria proposed, a Conference to Define Neuropathological Criteria for the Diagnosis of CTE sponsored by the National Institutes of Neurological Disorders and Stroke (NINDS) and the National Institute of Biomedical Imaging and Bioengineering (NIBIB) was held in 2015 (McKee et al. 2016). A panel of expert neuropathologists evaluated 25 cases of various tauopathies blinded to all clinical, demographic, and gross neuropathological information. The panel found that CTE was reliably distinguished from other tauopathies (including aging-related tau astrogliopathy [ARTAG]) (Kovacs et al. 2016) by the diagnostic criteria (McKee et al. 2016). In addition, the panel established that there was a pathognomonic lesion of CTE consisting of an abnormal accumulation of p-tau in neurons and astrocytes around small vessels irregularly distributed at the depths of the cortical sulci (McKee et al. 2016).

\section{CLINICAL SYNDROME}

In cases of neuropathologically confirmed CTE (McKee et al. 2013; Stein et al. 2015), the mean age at onset is 44.3 years $(\mathrm{SEM}=1.5$, range $=$ 16-83 years, $n=119$ ), the onset of clinical symptoms occurs an average of 14.5 years after retirement from the sport $(\mathrm{SEM}=1.6, n=$ 104 ), and $22 \%$ are symptomatic at the time of retirement. Moreover, the clinical course in CTE is prolonged (mean duration $=15.0$ years, $\mathrm{SEM}=1.2, n=125)$.

The clinical presentation of CTE consists of mood, behavioral, and cognitive alterations, as well as motor signs (Stern et al. 2013; Montenigro et al. 2014). Efforts to outline a consistent set of research and practical clinical diagnostic criteria have been proposed by several investigators (Jordan 2013; Stern et al. 2013; Victoroff 2013; Montenigro et al. 2014). Stern et al. (2013) proposed criteria for the diagnosis of 
the clinical syndrome associated with the pathology of CTE. They described two distinct clinical presentations of CTE: (1) a mood/behavior variant characterized by earlier onset (mean 35 years) and predominant features of explosivity, aggression, depression, apathy, and suicidality; and (2) a cognitive variant that usually presents later in life (mean 59 years) as memory deficits and executive dysfunction, which is very similar to $\mathrm{AD}$. A third mixed variant presenting as disturbances in both domains, with mood, behavior, and cognitive symptoms, was added by Montenigro et al. (2014). Dementia is common in advanced CTE, presenting occasionally with features of parkinsonism, dysarthia, dysphagia, and motor dysfunction (Stern et al. 2013; Montenigro et al. 2014).

\section{TRAUMATIC INJURY CASCADE LEADING TO CTE}

Axonal injury, microvascular injury, persistent neuroinflammation, and metabolic derangement that occur after repetitive subconcussion and concussion appear to trigger a pathological cascade that leads to CTE in susceptible individuals. Linear, and particularly rotational, acceleration-deceleration forces cause the semigelatinous brain to elongate and stretch; this distortion is greatest at the depths of the cerebral sulci of the brain and around the microvasculature. This tissue distortion produces multifocal injury to long filamentous structures in the brain, primarily the axons and small blood vessels, with multifocal blood-brain barrier loss. The time course for repair of the microstructural injuries is unclear, and there is evidence that some of the changes may be longstanding. Traumatic axonal injury results in alterations in axonal membrane permeability, ionic shifts including massive influx of calcium, and release of caspases and calpains. Traumatic injury is also associated with the hyperphosphorylation and mislocalization of the microtubule-associated protein tau. Under normal physiological conditions, tau is phosphorylated at only a small number of sites and is localized to the axon, where it binds to microtubules and stabi- lizes the cytoskeleton. Under pathological conditions, including after trauma, tau becomes hyperphosphorylated, dissociates from microtubules in the axon, and is displaced to the somatodendritic compartment as abnormal aggregates as NFTs (Binder et al. 2005; Serbest et al. 2007). Neuroinflammation, which occurs within minutes of a traumatic injury, can also promote tau pathology (Gyoneva et al. 2015). Cells at the injury site release cytokines and chemokines that lead to the recruitment of peripheral neutrophils and monocytes. At the same time, there is activation of resident astrocytes and microglia (Gyoneva et al. 2015). Acute metabolic disturbances also occur, including altered ionic flux, unregulated glutamate release, mitochondrial dysfunction, and oxidative stress (Giza and Hovda 2001). There is evidence that neurotoxic phosphorylated tau proteoforms aggregate early after the trauma and continue to accumulate and spread in the brain long after the traumatic insult (Kondo et al. 2015). Furthermore, these oligomeric, abnormally truncated, and phosphorylated tau proteins extensively co-localize in the perivascular tau lesions that are the essential, diagnostic hallmark of early CTE (Kanaan et al. 2016).

A unifying hypothesis in the pathogenesis of CTE would be that these abnormally phosphorylated tau proteoforms (protein molecules) accumulate at the stress points of the traumatic forces (i.e., at the depths of the cortical sulci and around small vessels). These isolated focal phosphorylated aggregates are associated with neurotoxicity and neurodegeneration and are pathologically evident as the pathognomonic lesions of CTE (stage I). Repetitive traumatic injury causes the production of additional perivascular lesions in multiple foci (stage II CTE). Cumulative posttraumatic accumulation of $\mathrm{p}$ tau proteoforms (phase I: trauma dependent) eventually builds to a critical threshold that overwhelms recovery mechanisms, and a runaway feed-forward neurodegenerative process (phase II: trauma independent) is set into motion. At this point, even in the absence of further trauma, the accumulation of p-tau aggregates has reached a level that promotes continued accumulation and spread through the nervous 
J. Kriegel et al.

system via a variety of mechanisms, possibly including protein templating or prion-like mechanisms of transneuronal and transsynaptic propagation (Lewis and Dickson 2016), tau secretion (Le et al. 2012), and extracellular cerebrospinal fluid (CSF) clearance pathways involving the glymphatic channels (Iliff et al. 2014). Neuroinflammation associated with the initial repetitive trauma and further aggravated by the accumulation of toxic p-tau fragments exacerbates neurodegeneration. This progressive neurodegeneration results in widespread $\mathrm{p}$-tau accumulation in cortical regions adjacent to the perivascular lesions, in the medial temporal lobe structures (hippocampus, amygdala, and entorhinal cortex) and in the deep nuclei (including the nucleus basalis of Meynert, substantia nigra, locus coeruleus, and others).

\section{TAU AS A PRION AND OTHER FACTORS THAT MIGHT EXPLAIN THE LATENT ONSET OF SYMPTOMS}

How tau pathology spreads through the brain in a disease-specific, stereotypical pattern is only beginning to be understood. Converging evidence from human, animal, and cultured cell studies argues that tau interacts with membrane components to facilitate a self-perpetuating prion-like propagation (Hall and Patuto 2012; Polymenidou and Cleveland 2012; Jucker and Walker 2013; Guo and Lee 2014; Lewis and Dickson 2016; see also Clavaguera et al. 2016; Rauch et al. 2016; Holmes and Diamond 2017). In this model, fibrillar tau aggregates are released into the extracellular space, where they are subsequently taken up by a recipient cell via macropinocytosis (Falcon et al. 2015). Direct contact with the recipient cell's natively folded tau protein results in templated misfolding and toxic amplification (Hall and Patuto 2012; Kfoury et al. 2012; Liu et al. 2012; Stancu et al. 2015; Clavaguera et al. 2016; Lewis and Dickson 2016). Indeed, wild-type mice injected with tau inclusions extracted from humans who died from various tauopathies (i.e., argyrophilic grain disease, progressive supranuclear palsy, corticobasal degeneration) develop pathologic lesions characteristic of the respective disease of origin (Clavaguera et al. 2013). The seeding potency of misfolded tau is dependent on its particular conformation and capacity to form aggregates (Falcon et al. 2015). In a self-perpetuating manner, tau pathology is spread along established networks of synaptic connectivity in the brain (Seeley et al. 2009). Although phosphorylation and misfolding of tau is initially a reversible process (Van der Jeugd et al. 2012; Wolozin 2012), tau seeding and propagation can promote further neuronal loss and neurodegeneration. The affected individual might be able to compensate for the pathology in the early stages, but progressive accumulation and neurodegeneration eventually produce clinical symptoms of disease.

Delayed onset of symptoms, often occurring decades after the trauma exposure, is characteristic of $80 \%$ of individuals with CTE (McKee et al. 2009). Factors that potentially influence the age at onset of symptoms might include disease location (i.e., perivascular $\mathrm{p}$ tau lesions in symptomatically sensitive regions, for example, amygdala, perirhinal cortex, prefrontal cortex, dorsolateral superior frontal cortex, etc.); differences in secondary modulating factors such as neuroinflammation; and resiliency factors such as cognitive reserve. Typically, if an individual becomes symptomatic with stage II CTE lesions, the symptoms that emerge are those of behavior and mood disorders. If the disease process develops more slowly, or perhaps if the early lesions do not involve symptomatically sensitive brain regions, or perhaps if an individual is able to compensate longer because of greater resiliency factors, symptoms do not appear until the disease has reached stage III when p-tau neurofibrillary degeneration involves widespread cortical and subcortical structures, including the hippocampus, amygdala, and entorhinal cortex, and becomes clinically evident as memory impairment and executive dysfunction. This would explain the age discrepancy between individuals who present with behavioral or mood disorders (stage II disease) (average age 35 years) and individuals who present with memory or executive dysfunction (stage III disease) (average age 54 years) (Stern et al. 2013). 


\section{BLOOD-BRAIN BARRIER DISRUPTION}

Other pathologies that contribute to CTE include microvascular injury and blood-brain barrier (BBB) disruption. The BBB is composed of a network of capillary endothelial cells joined by tight junctions and surrounded by basal lamina, pericytes, and astrocytic perivascular end feet. Astrocytes provide the cellular link to the neurons (Marchi et al. 2004). After a single season of play and in the absence of overt concussion, American football players were found to have imaging evidence of $\mathrm{BBB}$ disruption thought to be the result of exposure to "subconcussive" impacts (Marchi et al. 2013). BBB disruption has also been found after blast injury (Huber et al. 2016). In addition, $47 \%$ of late survivors of a single moderate to severe traumatic brain injury (TBI) were reported to have multifocal, abnormal, perivascular, and parenchymal fibrinogen and immunoglobulin $\mathrm{G}$ deposits in the cerebral cortex, suggesting that widespread BBB disruption may persist years after the traumatic insult (Hay et al. 2015).

Astrocytes produce the protein $\mathrm{S} 100 \beta$, which has been identified as a serum marker of BBB disruption (Fazio et al. 2004; Marchi et al. 2004). Although $S 100 \beta$ levels have been proposed as a method of detecting mild TBI in emergency rooms (Zongo et al. 2012), a study of 15 American football players who had played more than five games found that $S 100 \beta$ levels correlated with the frequency and severity of subconcussive hits (Marchi et al. 2013). Serum auto-S100 $\beta$ antibodies measured at the end of the season were also found to correlate with a player's cumulative single-game $S 100 \beta$ increases and the persistence of changes shown by diffusion tensor imaging. The results can be interpreted to suggest that repetitive breaches of the BBB, as reflected by serum $S 100 \beta$ levels, lead to a pathogenic autoimmune response directed against CNS antigens that have leaked through the BBB (Vincent et al. 2011; Marchi et al. 2013). Monocyte infiltration of the brain parenchyma via the leptomeninges also occurs after TBI. Acute TBI induces vascular damage, meningeal cell death, and the generation of reactive oxygen species (ROS) that ultimately breach the glial limitans and promote spread of the injury into the parenchyma. In response, the brain elicits a neuroprotective inflammatory response characterized by meningeal neutrophil swarming and microglial reconstitution of the damaged glial limitans (Roth et al. 2014). Activation of metalloproteinases produced by the infiltrating monocytes further aggravates the neuroinflammatory response and allows peripheral immune infiltration (Toft-Hansen et al. 2006; Newby 2008; Szmydynger-Chodobska et al. 2012).

\section{INFLAMMATORY RESPONSE}

Neuroinflammation has both beneficial and detrimental effects on the brain and spinal cord tissue (Jones et al. 2005; Block et al. 2007). Comprising $12 \%$ of cells in the brain, microglia represent the primary resident immune cells of the CNS (Block et al. 2007). In their usual resting state, microglia are highly dynamic and continuously survey and maintain the brain's microenvironment with motile processes and protuberances (Nimmerjahn et al. 2005). After TBI, microglia become more active, secreting proinflammatory cytokines, clearing debris or phagocytosing dead cells, and providing a cellular scaffolding to the area of injury. They then return to a resting state on resolution of the issue (Brockhaus et al. 1996; Norden et al. 2015). Significant heterogeneity with respect to motility and activity is observed in microglial populations following injury, which is likely attributable to highly localized signaling mechanisms (Petersen and Dailey 2004). In normal aging, as well as in neurodegeneration and following neurotrauma, microglia assume a rounder, shortened morphology and upregulate the expression of surface receptors, including the major histocompatibility complex (MHC) II (Cunningham et al. 2005; Frank et al. 2006; Henry et al. 2009; Lull and Block 2010; Norden and Godbout 2013; Fenn et al. 2014). In this primed state, microglia are hypervigilant in responding to environmental stressors. After injury, an exaggerated release of proinflammatory cytokines and chemokines, excitatory neurotransmitters, and reactive oxygen/nitrogen spe- 
J. Kriegel et al.

cies (ROS and RNS) ensues (Streit 2002). In some circumstances, including after minor neurotrauma, microglia become chronically activated with the development of an amoeboid or jellyfish-like morphology (Roth et al. 2014). Astrocytes express mechanosensitive ion channels, which, on release, activate a neuroprotective glial response to TBI. This response is followed shortly by astrocytic excretion of ATP, which activates resident microglia (Davalos et al. 2005) to form honeycomb networks that provide structural support to the injured meninges and parenchyma (Roth et al. 2014), as well as phagocytic jellyfish microglia to eliminate cellular debris. Concurrently, peripheral monocytes invade the brain parenchyma through the damaged meninges. This acute inflammatory reaction to brain injury appears to be beneficial and limits the extent of the injury (Finnie 2013).

\section{GLYMPHATIC SYSTEM-A PARAVASCULAR PATHWAY}

Although much is known about the lymphatic system and its role in clearing interstitial fluid (ISF) in the body, less is known about how the clearance of proteins and peptides is carried out in the brain, which lacks lymphatic vasculature. The CSF has long been thought to play a role in fluid exchange with the brain's ISF, serving as a sink for interstitial solute (Iliff et al. 2012). Iliff et al. (2012) found that the size of the radioactive tracer used correlated directly with the influx of CSF into the brain. Using in vivo laser microscopy techniques, they confirmed that subarachnoid CSF enters the parenchyma rapidly through paravascular spaces surrounding penetrating arteries. The fluid is subsequently cleared through large paravenous drainage pathways. Because of the significant role that glia cells, specifically astrocytes and the astroglial water channel aquaporin-4 (AQP4), play in the influx of CSF into the brain and the drainage of bulk ISF solute, they termed this the "glymphatic" system. Impairment of the glymphatic system in the TBI setting further appeared to promote neurofibrillary pathology and accumulation of exogenous tau around ves- sels (Iliff et al. 2014). This accumulation of tau around vessels is transient in mice; it remains to be determined whether disruption of the glymphatic flow contributes to the perivascular accumulation of tau in CTE.

\section{SUMMARY}

CTE is a latent neurodegeneration associated with repetitive concussive and subconcussive injury. There are often many years between exposure to brain trauma and the development of clinical symptoms of CTE. Pathologically, CTE begins as isolated perivascular foci of abnormally phosphorylated tau ( $\mathrm{p}$-tau) deposits in neurons and astrocytes in the cerebral cortex (stage I CTE). These initial CTE p-tau lesions accumulate at the stress points of the traumatic forces (i.e., at the depths of the cortical sulci and around small vessels and are associated with focal neurodegeneration). Repetitive traumatic injury causes the production of additional perivascular p-tau lesions in multiple foci (stage II CTE). Individuals with stage II CTE often show behavior and mood changes. It is possible that, at some point, the accumulation of p-tau aggregates reaches a level that promotes continued $\mathrm{p}$ tau accumulation and spread throughout the nervous system even in the absence of further exposure to trauma. This spread may involve protein templating mechanisms of transneuronal and transsynaptic propagation, tau secretion, and extracellular CSF clearance pathways involving the glymphatic channels. Neuroinflammation associated with the initial repetitive trauma and further aggravated by the accumulation of toxic $\mathrm{p}$-tau fragments might exacerbate neurodegeneration. As disease advances, hyperphosphorylated tau deposits are found in widespread cortical regions, as well as in the medial temporal lobe structures (hippocampus, amygdala, and entorhinal cortex) and in the deep nuclei (including the nucleus basalis of Meynert, substantia nigra, locus coeruleus, and others) (stage III CTE). Individuals with stage III CTE often experience memory loss and executive dysfunction. As p-tau aggregates continue to accumulate in widespread brain regions and neurodegeneration continues, there is incre- 
Is CTE a Prion Disease?

mental neuronal loss and p-tau accumulation in astrocytes (stage IV CTE). Individuals with stage IV CTE are frequently demented.

As CTE is a slowly progressive neurodegeneration, there is great hope for effective intervention early in the disease process before widespread neurodegeneration occurs. Effective intervention will require the development of methods to definitively detect focal CTE lesions during life when the initial perivascular aggregations of p-tau are just beginning to develop. Positron emission tomography using p-tau ligands and blood biomarker studies offer the greatest promise for this early in vivo detection, but to establish the required level of sensitivity and specificity will necessitate prospective clinical studies of individuals at high risk for CTE. Finally, there is great promise for the development of therapies to limit $\mathrm{p}$-tau propagation and spread-interventions that would interfere with the slow, relentless decline in subjects already exposed to the initial repetitive traumas and at high risk for CTE-induced neurodegeneration.

\section{REFERENCES}

${ }^{*}$ Reference is also in this collection.

Binder LI, Guillozet-Bongaarts AL, Garcia-Sierra F, Berry RW. 2005. Tau, tangles, and Alzheimer's disease. Biochim Biophys Acta 1739: 216-223.

Block ML, Zecca L, Hong JS. 2007. Microglia-mediated neurotoxicity: Uncovering the molecular mechanisms. Nat Rev Neurosci 8: 57-69.

Brandenburg W, Hallervorden J. 1954. Dementia pugilistica with anatomical findings. Virchows Arch Pathol Anat Physiol Klin Med 325: 680-709.

Brockhaus J, Möller T, Kettenmann H. 1996. Phagocytozing ameboid microglial cells studied in a mouse corpus callosum slice preparation. Glia 16: 81-90.

Clavaguera F, Akatsu H, Fraser G, Crowther RA, Frank S, Hench J, Probst A, Winkler DT, Reichwald J, Staufenbiel M. 2013. Brain homogenates from human tauopathies induce tau inclusions in mouse brain. Proc Natl Acad Sci 110: 9535-9540.

* Clavaguera F, Tolnay M, Goedert M. 2016. The prion-like behavior of assembled tau in transgenic mice. Cold Spring Harb Perspect Med doi: 10.1101/cshperspect. a024372.

Corsellis J, Bruton C, Freeman-Browne D. 1973. The aftermath of boxing. Psychol Med 3: 270-303.

Courville CB. 1962. Punch drunk. Its pathogenesis and pathology on the basis of a verified case. Bull Los Angel Neuro Soc 27: 160-168.
Cunningham C, Wilcockson DC, Campion S, Lunnon K, Perry VH. 2005. Central and systemic endotoxin challenges exacerbate the local inflammatory response and increase neuronal death during chronic neurodegeneration. J Neurosci 25: 9275-9284.

Davalos D, Grutzendler J, Yang G, Kim JV, Zuo Y, Jung S, Littman DR, Dustin ML, Gan WB. 2005. ATP mediates rapid microglial response to local brain injury in vivo. Nat Neurosci 8: 752-758.

Falcon B, Cavallini A, Angers R, Glover S, Murray TK, Barnham L, Jackson S, O’Neill MJ, Isaacs AM, Hutton ML. 2015. Conformation determines the seeding potencies of native and recombinant tau aggregates. J Biol Chem 290: 1049-1065.

Fazio V, Bhudia SK, Marchi N, Aumayr B, Janigro D. 2004. Peripheral detection of $\mathrm{S} 100 \beta$ during cardiothoracic surgery: What are we really measuring? Ann Thorac Surg 78: 46-52.

Fenn AM, Gensel JC, Huang Y, Popovich PG, Lifshitz J, Godbout JP. 2014. Immune activation promotes depression 1 month after diffuse brain injury: A role for primed microglia. Biol Psychiatry 76: 575-584.

Finnie J. 2013. Neuroinflammation: Beneficial and detrimental effects after traumatic brain injury. Inflammopharmacology 21: 309-320.

Frank MG, Barrientos RM, Biedenkapp JC, Rudy JW, Watkins LR, Maier SF. 2006. mRNA up-regulation of MHC II and pivotal pro-inflammatory genes in normal brain aging. Neurobiol Aging 27: 717-722.

Geddes JF, Vowles GH, Robinson SF, Sutcliffe JC. 1996. Neurofibrillary tangles, but not Alzheimer-type pathology, in a young boxer. Neuropathol Appl Neurobiol 22: 12-16.

Geddes J, Vowles G, Nicoll J, Revesz T. 1999. Neuronal cytoskeletal changes are an early consequence of repetitive head injury. Acta Neuropathol 98: 171-178.

Giza CC, Hovda DA. 2001. The neurometabolic cascade of concussion. J Athl Train 36: 228-235.

Goldstein LE, Fisher AM, Tagge CA, Zhang XL, Velisek L, Sullivan JA, Upreti C, Kracht JM, Ericsson M, Wojnarowicz MW, et al. 2012. Chronic traumatic encephalopathy in blast-exposed military veterans and a blast neurotrauma mouse model. Sci Transl Med 4: $134 \mathrm{ra} 60$.

Grahmann H, Ule G. 1957. Diagnosis of chronic cerebral symptoms in boxers (dementia pugilistica and traumatic encephalopathy of boxers). Psychiatr Neurol (Basel) 134: 261-283.

Guo JL, Lee VM. 2014. Cell-to-cell transmission of pathogenic proteins in neurodegenerative diseases. Nat Med 20: $130-138$.

Gyoneva S, Kim D, Katsumoto A, Kokiko-Cochran ON, Lamb BT, Ransohoff RM. 2015. Ccr2 deletion dissociates cavity size and tau pathology after mild traumatic brain injury. J Neuroinflammation 12: 228.

Hall GF, Patuto BA. 2012. Is tau ready for admission to the prion club? Prion 6: 223-233.

Hay JR, Johnson VE, Young AM, Smith DH, Stewart W. 2015. Blood-brain barrier disruption is an early event that may persist for many years after traumatic brain injury in humans. J Neuropathol Exp Neurol 74: 11471157. 
J. Kriegel et al.

Henry CJ, Huang Y, Wynne AM, Godbout JP. 2009. Peripheral lipopolysaccharide (LPS) challenge promotes microglial hyperactivity in aged mice that is associated with exaggerated induction of both pro-inflammatory IL- $1 \beta$ and anti-inflammatory IL-10 cytokines. Brain Behav Immun 23: 309-317.

Hof P, Knabe R, Bovier P, Bouras C. 1991. Neuropathological observations in a case of autism presenting with self-injury behavior. Acta Neuropathol 82: 321-326.

* Holmes BB, Diamond MI. 2017. Cellular models for the study of prions. Cold Spring Harb Perspect Med a024026.

Huber BR, Meabon JS, Hoffer ZS, Zhang J, Hoekstra JG, Pagulayan KF, McMillan PJ, Mayer CL, Banks WA, Kraemer BC. 2016. Blast exposure causes dynamic microglial/macrophage responses and microdomains of brain microvessel dysfunction. Neuroscience 319: 206-220.

Iliff JJ, Wang M, Liao Y, Plogg BA, Peng W, Gundersen GA, Benveniste H, Vates GE, Deane R, Goldman SA, et al. 2012. A paravascular pathway facilitates CSF flow through the brain parenchyma and the clearance of interstitial solutes, including amyloid $\beta$. Sci Trans Med 4 : $147 \mathrm{ral11.}$

Iliff JJ, Chen MJ, Plog BA, Zeppenfeld DM, Soltero M, Yang L, Singh I, Deane R, Nedergaard M. 2014. Impairment of glymphatic pathway function promotes tau pathology after traumatic brain injury. J Neurosci 34: 16180-16193.

Jones T, McDaniel E, Popovich P. 2005. Inflammatory-mediated injury and repair in the traumatically injured spinal cord. Curr Pharm Des 11: 1223-1236.

Jordan BD. 2013. The clinical spectrum of sport-related traumatic brain injury. Nat Rev Neurol 9: 222-230.

Jucker M, Walker LC. 2013. Self-propagation of pathogenic protein aggregates in neurodegenerative diseases. Nature 501: $45-51$.

Kanaan NM, Cox K, Alvarez VE, Stein TD, Poncil S, McKee AC. 2016. Characterization of early pathological tau conformations and phosphorylation in chronic traumatic encephalopathy. J Neuropathol Exp Neurol 75: 19-34.

Kfoury N, Holmes BB, Jiang H, Holtzman DM, Diamond MI. 2012. Trans-cellular propagation of Tau aggregation by fibrillar species. J Biol Chem 287: 19440-19451.

Kondo A, Shahpasand K, Mannix R, Qiu J, Moncaster J, Chen CH, Yao Y, Lin YM, Driver JA, Sun Y. 2015. Antibody against early driver of neurodegeneration cis P-tau blocks brain injury and tauopathy. Nature 523: 431-436.

Kovacs GG, Ferrer I, Grinberg LT, Alafuzoff I, Attems J, Budka H, Cairns NJ, Crary JF, Duyckaerts C, Ghetti B. 2016. Aging-related tau astrogliopathy (ARTAG): Harmonized evaluation strategy. Acta Neuropathol 131: 87102.

Le MN, Kim W, Lee S, McKee AC, Hall GF. 2012. Multiple mechanisms of extracellular tau spreading in a nontransgenic tauopathy model. Am J Neurodegener Dis 1: 316-333.

Lewis J, Dickson DW. 2016. Propagation of tau pathology: Hypotheses, discoveries, and yet unresolved questions from experimental and human brain studies. Acta Neuropathol 131: 27-48.

Liu L, Drouet V, Wu JW, Witter MP, Small SA, Clelland C, Duff K. 2012. Trans-synaptic spread of tau pathology in vivo. PLoS ONE 7: e31302.
Lull ME, Block ML. 2010. Microglial activation and chronic neurodegeneration. Neurotherapeutics 7: 354-365.

Marchi N, Cavaglia M, Fazio V, Bhudia S, Hallene K, Janigro D. 2004. Peripheral markers of blood-brain barrier damage. Clin Chim Acta 342: 1-12.

Marchi N, Bazarian JJ, Puvenna V, Janigro M, Ghosh C, Zhong J, Zhu T, Blackman E, Stewart D, Ellis J. 2013. Consequences of repeated blood-brain barrier disruption in football players. PLOS ONE 8: e56805.

Martland HS. 1928. Punch drunk. JAMA 91: 1103-1107.

Mawdsley C, Ferguson F. 1963. Neurological disease in boxers. Lancet 282: 795-801.

McKee AC, Robinson ME. 2014. Military-related traumatic brain injury and neurodegeneration. Alzheimers Dement 10: S242-S253.

McKee AC, Cantu RC, Nowinski CJ, Hedley-Whyte ET, Gavett BE, Budson AE, Santini VE, Lee HS, Kubilus CA, Stern RA. 2009. Chronic traumatic encephalopathy in athletes: Progressive tauopathy after repetitive head injury. J Neuropathol Exp Neurol 68: 709-735.

McKee AC, Gavett BE, Stern RA, Nowinski CJ, Cantu RC, Kowall NW, Perl DP, Hedley-Whyte ET, Price B, Sullivan C, et al. 2010. TDP-43 proteinopathy and motor neuron disease in chronic traumatic encephalopathy. J Neuropathol Exp Neurol 69: 918-929.

McKee AC, Stern RA, Nowinski CJ, Stein TD, Alvarez VE, Daneshvar DH, Lee HS, Wojtowicz SM, Hall G, Baugh $\mathrm{CM}$, et al. 2013. The spectrum of disease in chronic traumatic encephalopathy. Brain 136: 43-64.

McKee AC, Daneshvar DH, Alvarez VE, Stein TD. 2014. The neuropathology of sport. Acta Neuropathol 127: 29-51.

McKee AC, Stein TD, Kiernan PT, Alvarez VE. 2015. The neuropathology of chronic traumatic encephalopathy. Brain Pathol 25: 350-364.

McKee AC, Cairns NJ, Dickson DW, Folkerth RD, Keene CD, Litvan I, Perl DP, Stein TD, Vonsattel JP, Stewart W. 2016. The first NINDS/NIBIB consensus meeting to define neuropathological criteria for the diagnosis of chronic traumatic encephalopathy. Acta Neuropathol 131: 75-86.

Mez J, Solomon TM, Daneshvar DH, Murphy L, Kiernan PT, Montenigro PH, Kriegel J, Abdolmohammadi B, Fry B, Babcock KJ. 2015. Assessing clinicopathological correlation in chronic traumatic encephalopathy: Rationale and methods for the UNITE study. Alzheimers Res Ther 7: $1-14$.

Millspaugh J. 1937. Dementia pugilistica. US Naval Med Bull 35: 297-303.

Montenigro PH, Baugh CM, Daneshvar DH, Mez J, Budson AE, Au R, Katz DI, Cantu RC, Stern RA. 2014. Clinical subtypes of chronic traumatic encephalopathy: Literature review and proposed research diagnostic criteria for traumatic encephalopathy syndrome. Alzheimers Res Ther 6: $1-17$.

Neubuerger KT, Sinton DW, Denst J. 1959. Cerebral atrophy associated with boxing. AMA Arch Neurol Psychiatry 81: 403-408.

Newby AC. 2008. Metalloproteinase expression in monocytes and macrophages and its relationship to atherosclerotic plaque instability. Arterioscler Thromb Vasc Biol 28: 2108-2114. 
Is CTE a Prion Disease?

Nimmerjahn A, Kirchhoff F, Helmchen F. 2005. Resting microglial cells are highly dynamic surveillants of brain parenchyma in vivo. Science 308: 1314-1318.

Norden DM, Godbout JP. 2013. Review: Microglia of the aged brain: Primed to be activated and resistant to regulation. Neuropathol Appl Neurobiol 39: 19-34.

Norden DM, Muccigrosso MM, Godbout JP. 2015. Microglial priming and enhanced reactivity to secondary insult in aging, and traumatic CNS injury, and neurodegenerative disease. Neuropharmacology 96: 29-41.

Omalu B, Hammers JL, Bailes J, Hamilton RL, Kamboh MI, Webster G, Fitzsimmons RP. 2011. Chronic traumatic encephalopathy in an Iraqi war veteran with posttraumatic stress disorder who committed suicide. Neurosurg Focus 31: E3.

Payne E. 1968. Brains of boxers. Neurochirurgia 11: 173188.

Petersen MA, Dailey ME. 2004. Diverse microglial motility behaviors during clearance of dead cells in hippocampal slices. Glia 46: 195-206.

Polymenidou M, Cleveland DW. 2012. Prion-like spread of protein aggregates in neurodegeneration. J Exp Med 209: 889-893.

* Rauch JN, Olson SH, Gestwicki JE. 2016. Interactions between microtubule-associated protein tau (MAPT) and small molecules. Cold Spring Harb Perspect Med doi: 10.1101/cshperspect.a024034.

Roth TL, Nayak D, Atanasijevic T, Koretsky AP, Latour LL, McGavern DB. 2014. Transcranial amelioration of inflammation and cell death after brain injury. Nature 505: $223-228$.

Seeley WW, Crawford RK, Zhou J, Miller BL, Greicius MD. 2009. Neurodegenerative diseases target large-scale human brain networks. Neuron 62: 42-52.

Serbest G, Burkhardt MF, Siman R, Raghupathi R, Saatman KE. 2007. Temporal profiles of cytoskeletal protein loss following traumatic axonal injury in mice. Neurochem Res 32: 2006-2014.

Stancu IC, Vasconcelos B, Ris L, Wang P, Villers A, Peeraer E, Buist A, Terwel D, Baatsen P, Oyelami T. 2015. Templated misfolding of Tau by prion-like seeding along neuronal connections impairs neuronal network function and associated behavioral outcomes in Tau transgenic mice. Acta Neuropathol 129: 875-894.

Stein TD, Montenigro PH, Alvarez VE, Xia W, Crary JF, Tripodis Y, Daneshvar DH, Mez J, Solomon T, Meng G, et al. 2015. $\beta$-Amyloid deposition in chronic traumatic encephalopathy. Acta Neuropathol 130: 21-34.

Stern RA, Daneshvar DH, Baugh CM, Seichepine DR, Montenigro PH, Riley DO, Fritts NG, Stamm JM, Robbins CA, McHale L. 2013. Clinical presentation of chronic traumatic encephalopathy. Neurology 81: 1122-1129.

Streit WJ. 2002. Microglia as neuroprotective, immunocompetent cells of the CNS. Glia 40: 133-139.

Szmydynger-Chodobska J, Strazielle N, Gandy JR, Keefe TH, Zink BJ, Ghersi-Egea JF, Chodobski A. 2012. Posttraumatic invasion of monocytes across the blood-cerebrospinal fluid barrier. J Cereb Blood Flow Metab 32: 93-104.

Toft-Hansen H, Buist R, Sun XJ, Schellenberg A, Peeling J, Owens T. 2006. Metalloproteinases control brain inflammation induced by pertussis toxin in mice overexpressing the chemokine CCL2 in the central nervous system. J Immunol 177: 7242-7249.

Van der Jeugd A, Hochgräfe K, Ahmed T, Decker JM, Sydow A, Hofmann A, Wu D, Messing L, Balschun D, D’Hooge R. 2012. Cognitive defects are reversible in inducible mice expressing pro-aggregant full-length human Tau. Acto Neuropathol 123: 787-805.

Victoroff J. 2013. Traumatic encephalopathy: Review and provisional research diagnostic criteria. NeuroRehabilitation 32: 211-224.

Vincent A, Bien CG, Irani SR, Waters P. 2011. Autoantibodies associated with diseases of the CNS: New developments and future challenges. Lancet Neurol 10: 759-772.

Wolozin B. 2012. Regulated protein aggregation: Stress granules and neurodegeneration. Mol Neurodegener 7: 56-56.

Zongo D, Ribéreau-Gayon R, Masson F, Laborey M, Contrand B, Salmi LR, Montaudon D, Beaudeux JL, Meurin A, Dousset V. 2012. S100-B protein as a screening tool for the early assessment of minor head injury. Ann Emerg Med 59: 209-218. 


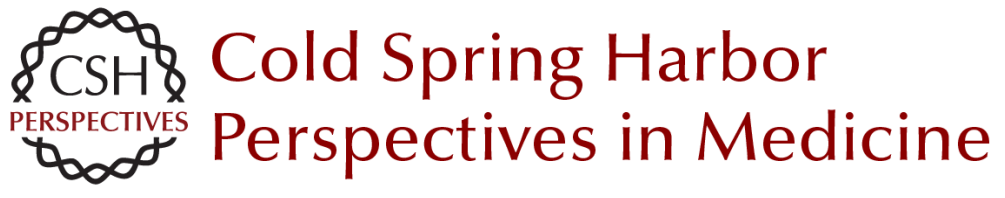

\section{Chronic Traumatic Encephalopathy: Is Latency in Symptom Onset Explained by Tau Propagation?}

Joshua Kriegel, Zachary Papadopoulos and Ann C. McKee

Cold Spring Harb Perspect Med 2018; doi: 10.1101/cshperspect.a024059 originally published online January 17, 2017

\section{Subject Collection Prion Diseases}

TDP-43 Prions

Takashi Nonaka and Masato Hasegawa

$\alpha$-Synuclein: Multiple System Atrophy Prions

Amanda L. Woerman, Joel C. Watts, Atsushi

Aoyagi, et al.

Genetics of Synucleinopathies

Robert L. Nussbaum

$\beta$-Amyloid Prions and the Pathobiology of

Alzheimer's Disease Joel C. Watts and Stanley B. Prusiner

Disease Mechanisms of C9ORF72 Repeat

Expansions

Tania F. Gendron and Leonard Petrucelli

Chronic Traumatic Encephalopathy: Is Latency in

Symptom Onset Explained by Tau Propagation? Joshua Kriegel, Zachary Papadopoulos and Ann C. McKee

Noncerebral Amyloidoses: Aspects on Seeding, Cross-Seeding, and Transmission

Gunilla T. Westermark, Marcus Fändrich, Katarzyna Lundmark, et al.

Structural and Chemical Biology of Presenilin Complexes

Douglas S. Johnson, Yue-Ming Li, Martin

Pettersson, et al.
Cell Biology and Pathophysiology of $\alpha$-Synuclein Jacqueline Burré, Manu Sharma and Thomas C. Südhof

Molecular Mechanisms of Chronic Wasting

Disease Prion Propagation Julie A. Moreno and Glenn C. Telling

Genetics of Amyotrophic Lateral Sclerosis Mehdi Ghasemi and Robert H. Brown, Jr.

The Genetics of C9orf72 Expansions Ilse Gijselinck, Marc Cruts and Christine Van Broeckhoven

Prion-Like Characteristics of

Polyglutamine-Containing Proteins Margaret M.P. Pearce and Ron R. Kopito

Therapeutic Strategies for Restoring Tau Homeostasis

Zapporah T. Young, Sue Ann Mok and Jason E. Gestwicki

Fused in Sarcoma Neuropathology in Neurodegenerative Disease

Ian R.A. Mackenzie and Manuela Neumann

Experimental Models of Inherited PrP Prion

Diseases

Joel C. Watts and Stanley B. Prusiner

For additional articles in this collection, see http://perspectivesinmedicine.cshlp.org/cgi/collection/ 